\title{
Towards a 3D likelihood analysis in very-high-energy $\gamma$-ray astronomy: the case of H.E.S.S.
}

\author{
Lars Mohrmann; Kaori Nakashima, Andreas Specovius, Domenico Tiziani, Stefan \\ Funk, Christopher van Eldik \\ Erlangen Centre for Astroparticle Physics, Erwin-Rommel-Str. 1, 91058 Erlangen, Germany \\ E-mail: lars.mohrmann@fau.de
}

\section{Stefan Ohm ${ }^{\dagger}$}

DESY, Platanenallee 6, 15738 Zeuthen, Germany

\begin{abstract}
The estimation of the residual background due to cosmic ray-induced air showers represents a key element of every analysis of Imaging Atmospheric Cherenkov Telescope (IACT) data. In the past, most techniques have relied on a measurement of the background in supposedly sourcefree regions within the observed field of view. However, this approach is problematic in regions with many (potentially overlapping) $\gamma$-ray sources or large-scale diffuse $\gamma$-ray emission, like e.g. the Galactic Plane. For these cases, a template-based 3D likelihood analysis might be superior. In this approach, a spectro-morphological model template, consisting of one or multiple source components and a background component, is fit to the data, resulting in a complete spectral and spatial description of the field of view. In this contribution, we present our efforts to establish a 3D likelihood analysis for data taken with the H.E.S.S. system of IACTs, using the open-source analysis packages ctools and Gammapy. In particular, we focus on the development of a template model for the cosmic ray-induced background - a major challenge in such an approach - from archival H.E.S.S. data. We characterise the obtained model and demonstrate its application using publicly available H.E.S.S. data. The validation of this analysis approach on experimental data also constitutes an important step towards the analysis of data from the upcoming Cherenkov Telescope Array (CTA).
\end{abstract}

36th International Cosmic Ray Conference -ICRC2019-

July 24th - August 1st, 2019

Madison, WI, U.S.A.

\footnotetext{
${ }^{*}$ Contact author

$\dagger$ Presenter
} 


\section{Introduction}

With its Galactic Plane Survey, the H.E.S.S. Collaboration has carried out an extensive observation program of the inner Galaxy in very-high-energy $\gamma$ rays [1]. A plethora of VHE $\gamma$-ray sources has been discovered, many of them extended, covering up to more than a square degree on the sky. Additionally, large-scale diffuse $\gamma$-ray emission has been detected with H.E.S.S. in the Galactic Plane $[2,3]$. This implies that a proper description of many regions within the Galactic Plane can only be obtained by modeling multiple, possibly overlapping components, a situation that will become even more severe for the upcoming, much more sensitive Cherenkov Telescope Array (CTA) [4].

Traditional methods for the analysis of Imaging Atmospheric Cherenkov Telescope (IACT) data do not cope well with this situation. In particular, these methods often rely on a measurement of the residual, cosmic ray-induced background in the observed field of view, thus requiring regions free of $\gamma$-ray emission (see [5] for an overview of standard methods). Here, we demonstrate a new analysis approach that is better suited for the analysis of complicated fields of view, namely a 3D likelihood analysis based on model templates. In this approach, three-dimensional spectro-morphological templates for all relevant components are fitted to the data, thus achieving a complete description of the observed field of view. One major challenge is the preparation of an accurate model for the residual cosmic ray-induced background, which strongly depends on the observation conditions.

In Section 2, we introduce a procedure to construct such a background model, based on archival H.E.S.S. data. We characterise its principal features and perform a general validation. Then, in Section 3, we utilise the background model to apply the 3D likelihood analysis to a public H.E.S.S. test data set [6]. The analysis is carried out using ctools [7] and Gammapy [8], two software packages that are proposed as high-level data analysis tools for CTA.

This contribution is based on [9], to which the reader is referred for a more detailed account.

\section{Background Model}

We construct a model that predicts the rate of cosmic-ray background events for arbitrary observations, as a function of two spatial coordinates and the reconstructed energy of the primary particle. The procedure was pioneered by [10], but has been considerably refined in this work.

\subsection{Construction}

The background model is constructed from archival H.E.S.S. data. Here, we restrict ourselves to data taken with the first phase of the H.E.S.S. experiment, when it consisted of four Cherenkov telescopes with $107 \mathrm{~m}^{2}$ mirror area each. We exclude observations taken in the direction of the Galactic plane $\left(|l|<60^{\circ},|b|<5^{\circ}\right)$, to avoid contamination by unresolved $\gamma$-ray sources or diffuse $\gamma$ ray emission. Furthermore, we use only observations fulfilling "spectral" quality, indicating stable hardware and good atmospheric conditions, and only observations taken with all four telescopes. In total, this yields 7063 observations with a total observation time of $\approx 3240$ hours, taken between January 21, 2004 and May 15, 2013. 
The construction procedure is illustrated in Fig. 1. We construct the model in a field-of-view coordinate system, in which the pointing direction of the telescopes is centred at $(x=0, y=0)$, and whose axes are aligned with the altitude-azimuth system. Event coordinates are transformed into this system and the predicted background rate is obtained by averaging over multiple observations. Events registered from regions containing known $\gamma$-ray emitters are not considered, and a corresponding correction is performed for the exposure time of each pixel.

The background rate strongly depends on the observation conditions, most notably the zenith angle of the pointing direction of the observation. A less pronounced dependence on the azimuth angle, due to the Earth's magnetic field, is also expected. We therefore divide the observations into bins of zenith and azimuth angle and construct a model for each of these bins.

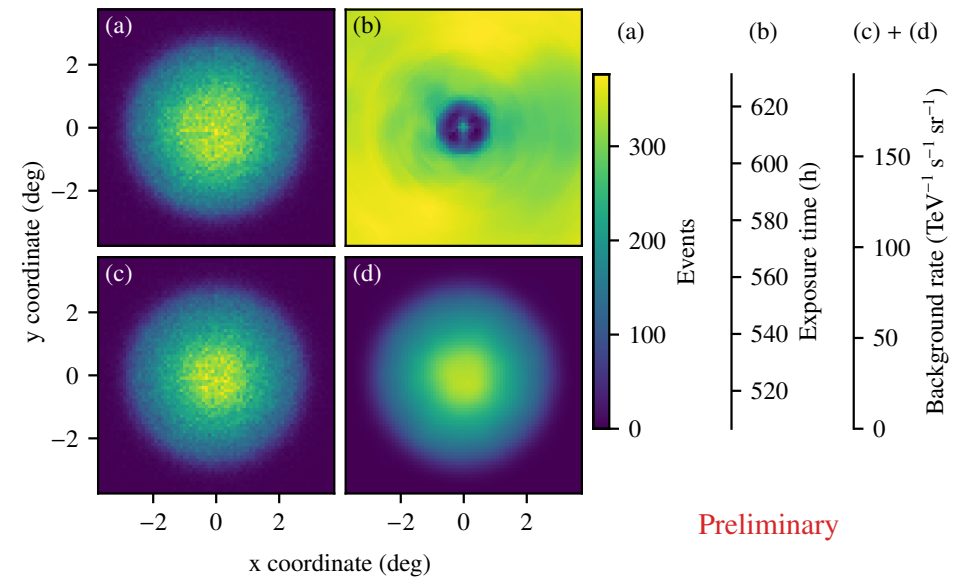

Figure 1: Illustration of the construction of the background model, shown here for the bin with azimuth angle $90^{\circ}<\phi<270^{\circ}$ and zenith angle $20^{\circ}<\vartheta<30^{\circ}$ and energy $0.8 \mathrm{TeV}<E<1.1 \mathrm{TeV}$. (a) Sum of the number of registered events in each observation, excluding events with a reconstructed direction close to known $\gamma$-ray sources. (b) Effective exposure time of each spatial pixel. The effective exposure time is the summed observation time of all observations, corrected for the exclusion of events from regions around known $\gamma$-ray sources. (c) Averaged background rate, given by the number of registered events divided by effective exposure time, energy interval and solid angle. (d) Averaged background rate after the application of a smoothing algorithm. Note that all vertical axis labels refer to the same color bar.

In a second step, the background model is evaluated for individual observations. To better capture the dependence on the zenith angle, we perform an interpolation between the zenith angle bins. Furthermore, we apply corrections for the atmospheric transparency, using the so-called transparency coefficient (TC) [11], and for the optical efficiency of the telescopes measured for the observation in question. For a more detailed description of the background model construction procedure, please refer to [9].

\subsection{Validation}

In order to validate the background model, we have performed likelihood fits of the model to all H.E.S.S. observations taken in the first phase of the H.E.S.S. experiment. We introduced two parameters that were optimised in the fit: the normalisation of the background model, and a spectral "tilt", which modifies the predicted rate $R$ at energy $E$ as $R^{\prime}=R \cdot\left(E / E_{0}\right)^{-\delta}$, where $\delta$ is 
the tilt parameter. These are the same parameters that are also available for the background model in the 3D likelihood analysis with ctools and Gammapy. Regions containing known $\gamma$-ray sources were excluded from the fit.

Figure 2 shows distributions of the two parameters for all 4-telescope observations, and for those observations that have been used in the construction of the model. For the latter, we observe that the rate of cosmic-ray background events is predicted by the model with a spread of 9\%. Expectedly, the spread increases to $13 \%$ when all observations are considered, i.e. also those affected by unresolved $\gamma$-ray sources, diffuse $\gamma$-ray emission, or bad atmospheric conditions. The relatively small bias and small spread for the spectral tilt parameter furthermore illustrates that also the spectral shape of the cosmic-ray background is described well by our model.

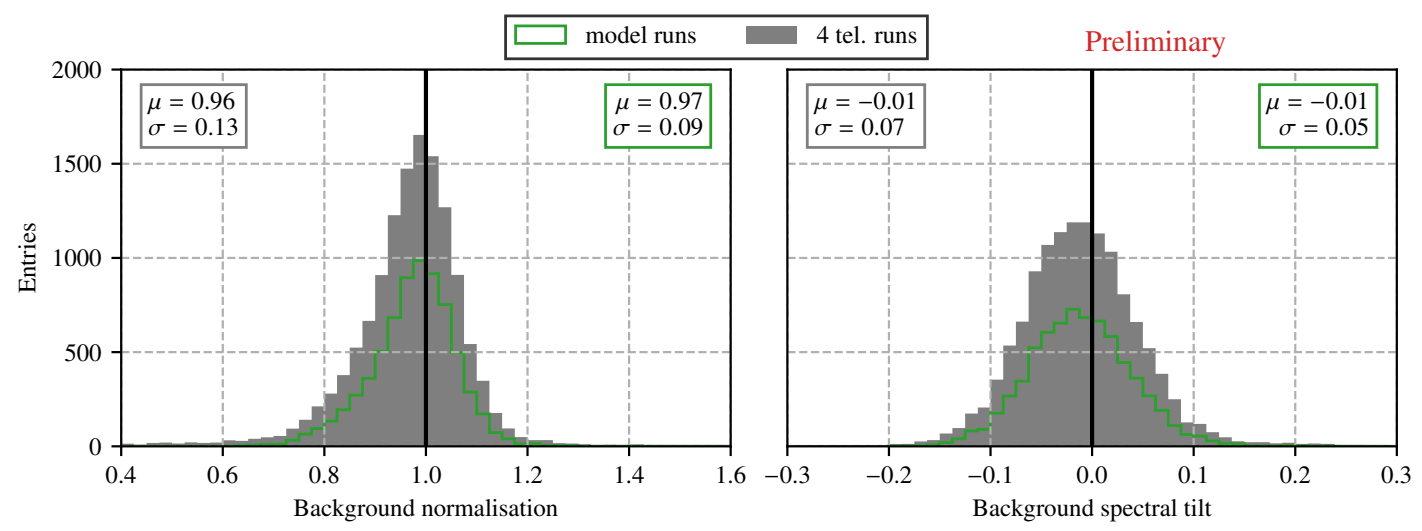

Figure 2: Distribution of fitted background model normalisations and spectral tilts. The grey, filled histograms are for all 4-telescope observations, while the green histograms contain only those observations used in the construction of the model. The mean value $(\mu)$ and standard deviation $(\sigma)$ of the distributions are given as well.

In order to also assess the quality of the description of the spatial shape of the background by the model, we consider energy-integrated slices of the data along the right-ascension or declination axis. A slice along the right-ascension axis is shown for one example observation in Figure 3. The data are described well by the background model in this case, as also indicated by a $\chi^{2}$ test statistic of 48.0 , for 44 degrees of freedom. We have performed a $\chi^{2}$ test for slices along both spatial axes for all observations. In the left panels of Fig. 4, we show distributions of the resulting $\chi^{2}$ probabilities, again separated for all 4-telescope observations and those observations used in the model construction. These distributions are expected to be flat for a perfect description of the data by the background model. The right panels show the corresponding $\chi^{2}$ significance values in terms of a normal distribution. Here, a Gaussian distribution with zero mean and unity width is expected. Overall, we conclude that also the spatial shape of the background rate is described well for most of the observations by our model.

\section{3D Likelihood Analysis}

In this section, we employ the developed cosmic-ray background model in a 3D likelihood analysis performed on public H.E.S.S. data [6]. We show exemplary results obtained on two well- 


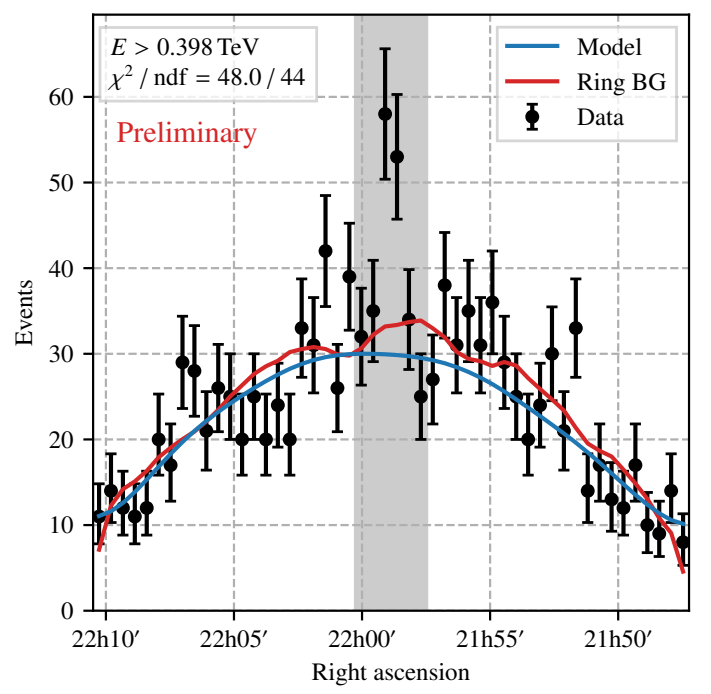

Figure 3: Number of events for one example observation, as a function of right-ascension (J2000). Shown are all events with declination $\left|\delta-\delta_{\mathrm{pnt}}\right|<0.7 \mathrm{deg}$, where $\delta_{\mathrm{pnt}}$ is the declination of the pointing position. The grey-shaded area marks an exclusion region around the source position (PKS 2155-304 in this case). A background estimate obtained with the ring background method [5] is shown as well.

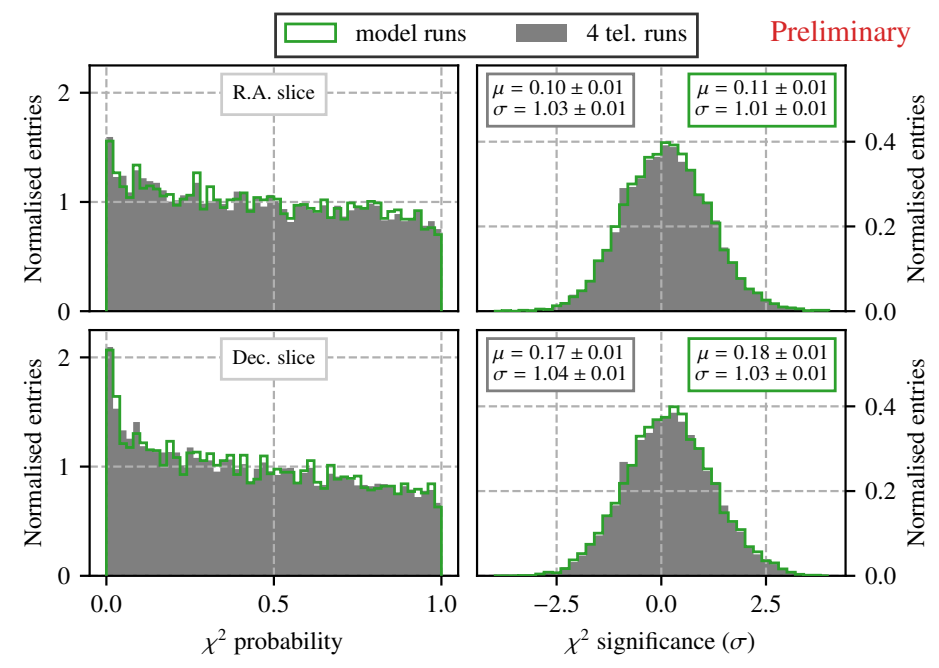

Figure 4: Distribution of $\chi^{2}$ probabilities and significance values, for slices along right-ascension (top) and declination (bottom). The grey, filled histograms are for all 4-telescope observations, while the green histograms contain only those observations used in the construction of the model.

known $\gamma$-ray sources, the pulsar wind nebula MSH 15-52 [12] and the shell-type supernova remnant RX J1713.7-3946 [13]. We use a simple power law as spectral model for both sources.

The morphology of MSH 15-52 is simple enough such that it can be modelled using an analytical model, here we use an elliptical disk model. In Fig. 5(a), we show a residual significance map for the best-fit model. The map shows no features except for statistical fluctuations, indicating that both the source as well as the cosmic-ray background are described well by our models.

In contrast, the complex morphology of RX J1713.7-3946 requires us to use a template map 
as spatial model for the source. Here, we obtain such a template map from the data themselves by first performing a fit of only the cosmic-ray background model to the data, excluding the source region from the fit. Subtracting the best-fit background model from the data yields a map of excess events from the source, which we smooth to obtain a template map for RX J1713.7-3946 in the full fit. The best-fit model (background + source template) is shown together with the observed data in Fig. 6. The residual significance map for RX J1713.7-3946 is shown in Fig. 5(b).

The significance map for RX J1713.7-3946 shows two notable features: (i) a residual positive excess to the south-east of the source; this could be caused by a true excess of $\gamma$ rays e.g. from unresolved sources, or by an imperfect background model. (ii) the region covered by the source template is almost free of statistical fluctuations; this is an artifact of the generation of the template map, which is created from the data themselves and thus - despite the applied smoothing - subject to the same statistical fluctuations.

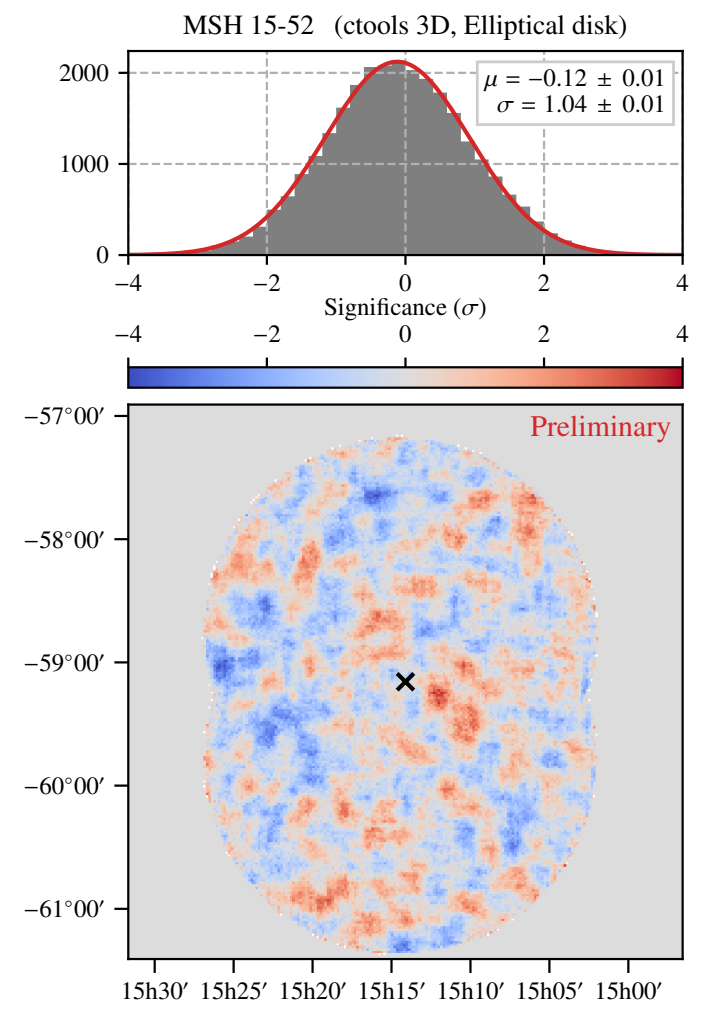

(a) MSH 15-52. Obtained with ctools.

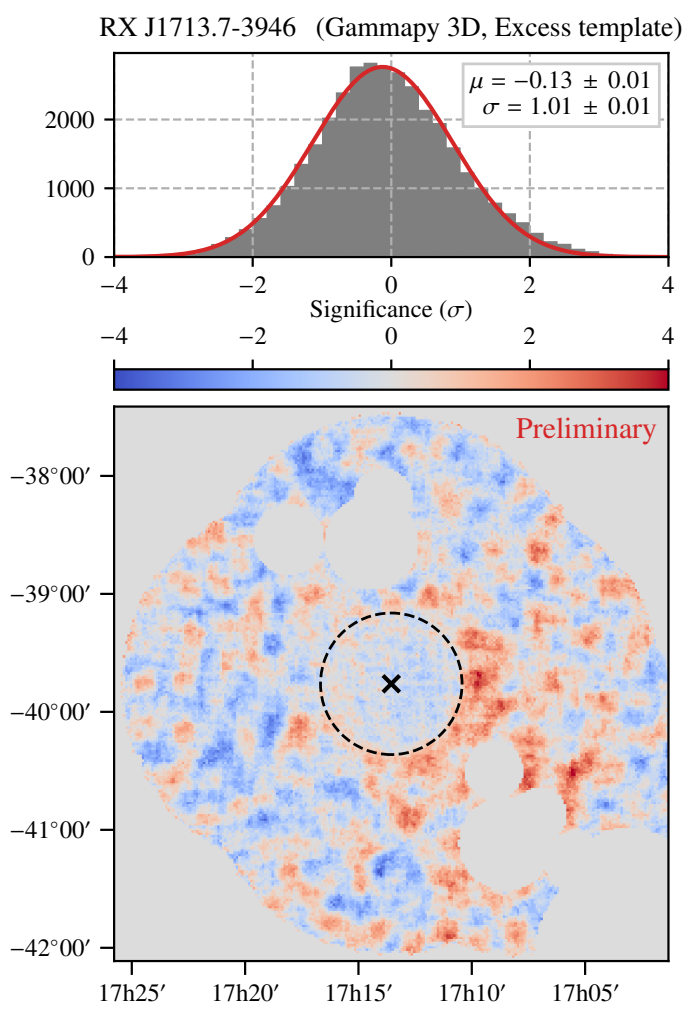

(b) RX J1713.7-3946. Obtained with Gammapy.

Figure 5: Residual significance maps, in equatorial coordinates (J2000). Grey-filled areas have been excluded from the fit. The dashed line indicates the size of the source template employed for RX J1713.7-3946. The top panels show the corresponding entry distributions, with their me and spread.

Finally, we show the obtained spectra for MSH 15-52 and RX J1713.7-3946 in Figs. 7(a) and 7(b), respectively. The results are compared to those obtained with the H.E.S.S.-internal analysis program $H A P$, which were derived with the same data set using the reflected background method, a well-established analysis technique in IACT data analysis [5]. We observe a very good overall agreement. The only notable deviation is between the spectra for RX J1713.7-3946 derived 


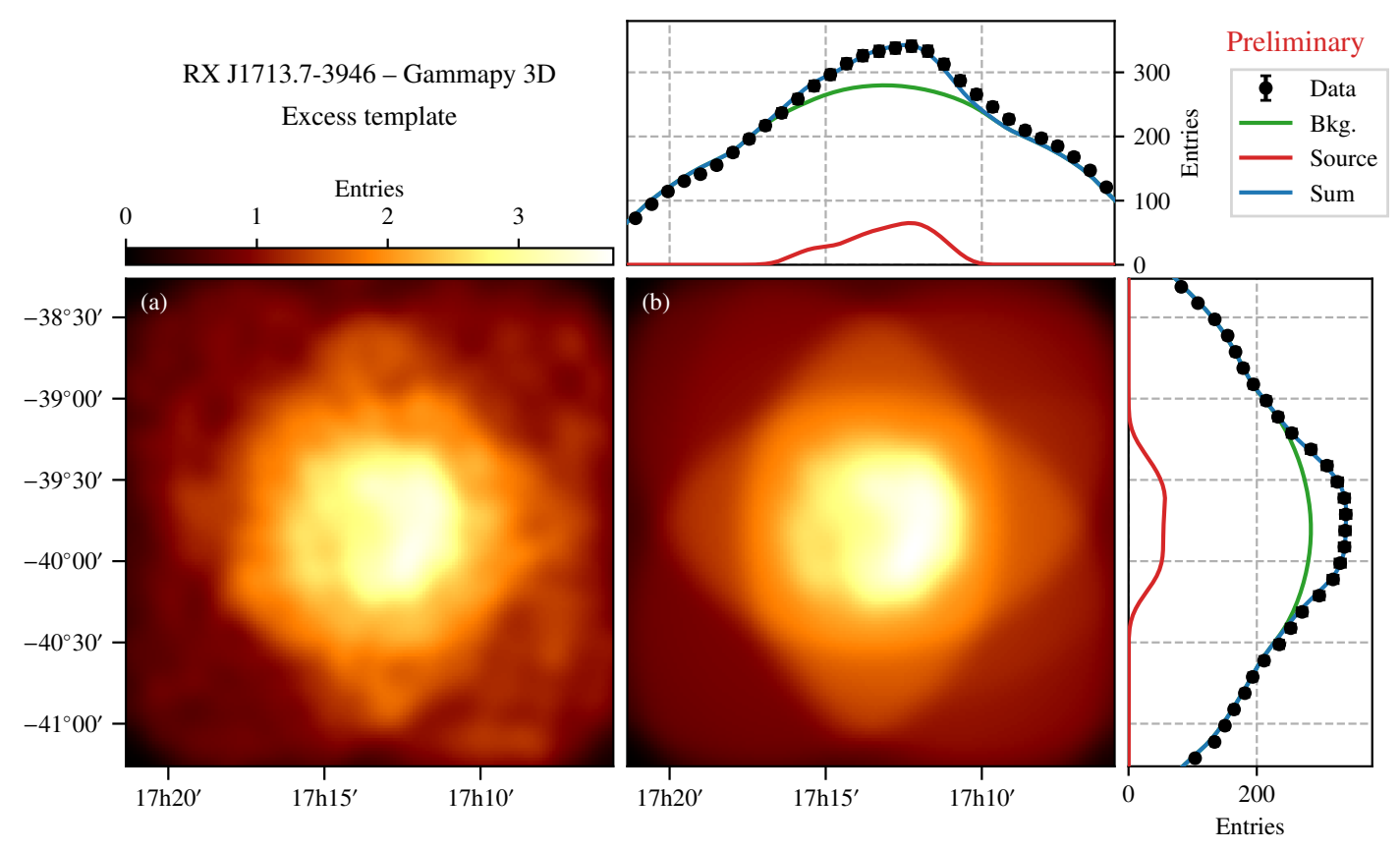

Figure 6: Data (a) and model (b) maps for RX J1713.7-3946, in equatorial coordinates (J2000), obtained with Gammapy. The small panels show projections onto the right-ascension and declination axes.

here (with ctools, Gammapy and HAP) and the spectrum published in [13]. That the results obtained here agree well between the tools indicates that the discrepancy is likely due to an issue with the analysed data set (which is much smaller than the one used in [13]).

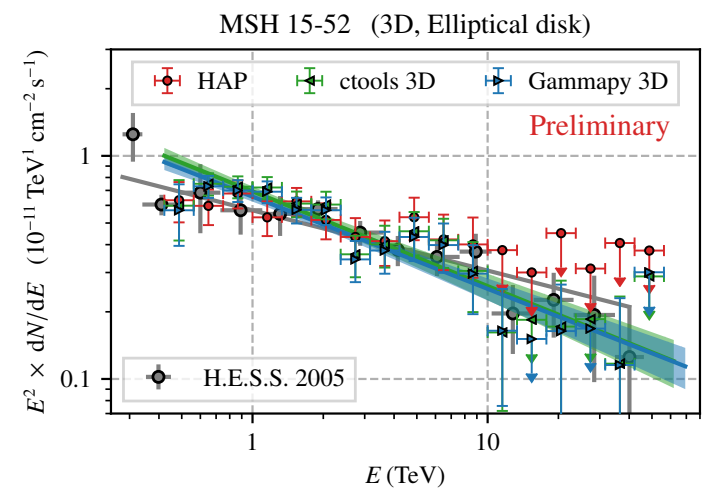

(a) MSH 15-52. Published spectrum from [12].

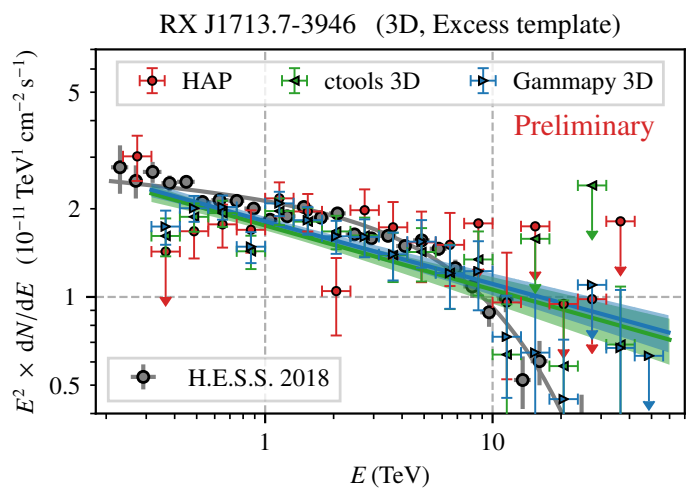

(b) RX J1713.7-3946. Published spectrum from [13].

Figure 7: Spectra obtained for MSH 15-52 and RX J1713.7-3946. Results are compared to those obtained with the H.E.S.S.-internal analysis tool HAP.

\section{Conclusion}

We have presented a procedure for the construction of a three-dimensional model for the cosmic-ray background in the H.E.S.S. experiment. A validation of the model on many observa- 
tions has shown that it is able to describe both the spatial and the spectral shape of the cosmic-ray background well.

We have then employed this model in a 3D likelihood analysis carried out on a public H.E.S.S. test data set. We obtained results that are compatible with those obtained with standard techniques as well as with published results; this validates both the background model and the analysis approach. This result is important also for the upcoming CTA, where a 3D likelihood analysis will be required in many cases due to the largely increased sensitivity.

\section{Acknowledgments}

We thank the H.E.S.S. Collaboration for allowing us to use their internal analysis software package and their archival data. We acknowledge helpful support from the developer teams of the ctools and Gammapy packages.

\section{References}

[1] H. Abdalla et al., The H.E.S.S. Galactic plane survey, A\&A 612, A1 (2018).

[2] A. Abramowski et al., Diffuse Galactic gamma-ray emission with H.E.S.S., Phys. Rev. D 90, 122007 (2014).

[3] H. Abdalla et al., Characterising the VHE diffuse emission in the central 200 parsecs of our Galaxy with H.E.S.S., A\&A 612, A9 (2018).

[4] D. Mazin et al., The Cherenkov Telescope Array, in: Proc. 36th Int. Cosmic Ray Conf., POS (ICRC2019) 741 (2019).

[5] D. Berge, S. Funk, and J. Hinton, Background modelling in very-high-energy $\gamma$-ray astronomy, A\&A 466, 1219 (2007).

[6] H. Abdalla et al., H.E.S.S. first public test data release, arXiv:1810.04516, https://zenodo.org/record/1421099 (2018).

[7] J. Knödlseder et. al., GammaLib and ctools: A software framework for the analysis of astronomical gamma-ray data, A\&A 593, A1 (2016).

[8] C. Deil et al., Gammapy - A prototype for the CTA science tools, in: Proc. 35th Int. Cosmic Ray Conf., POS ( ICRC2017) 766 (2017).

[9] L. Mohrmann et al., Open-source science tools in very-high-energy $\gamma$-ray astronomy: background model construction for template analysis and tool validation using public H.E.S.S. data, to be submitted to A\&A (2019).

[10] M. Mayer, Pulsar wind nebulae at high energies: A Diverse Population and Exceptional Twins, $\mathrm{PhD}$ thesis, Potsdam University (2014).

[11] J. Hahn et al., Impact of aerosols and adverse atmospheric conditions on the data quality for spectral analysis of the H.E.S.S. telescopes, Astropart. Phys. 54, 25-32 (2014).

[12] F. Aharonian et al., Discovery of extended VHE gamma-ray emission from the asymmetric pulsar wind nebula in MSH 15-52 with HESS, A\&A 435, L17 (2005).

[13] H. Abdalla et al., H.E.S.S. observations of RX J1713.7-3946 with improved angular and spectral resolution: Evidence for gamma-ray emission extending beyond the X-ray emitting shell, A\&A $\mathbf{6 1 2}$, A6 (2018). 